\title{
Invariant Unstable Manifolds of Nonautonomous Systems on Time Scales
}

\author{
Le Huy Tien ${ }^{1}$, Nguyen Minh Man $^{2}$ and Le Duc Nhien ${ }^{1 *}$ \\ ${ }^{1}$ Department of Mathematics, Mechanics and Informatics, Vietnam National University at Hanoi, 334 Nguyen \\ Trai, Thanh Xuan, Hanoi, Viet Nam \\ ${ }^{2}$ Faculty of Basic Sciences, Ha Noi University of Mining and Geology, 18 Pho Vien, Ha Noi, Viet Nam \\ Email: nhien0610@gmail.com
}

\begin{abstract}
This paper is concerned with the existence of invariant manifolds for dynamical equations on a periodic time scale when the nonlinear perturbation has a small global Lipschitz constant. Particularly, for time-varying non-regressive dynamical equations, which have exponential dichotomies on a periodic time scale with bounded graininess, we use the method of graph transforms as in [1] to prove that there exists a unique integral manifold of that systems.
\end{abstract}

Keywords: Integral manifold, graph transforms, time scales, linear dynamic equation.

\section{Introduction and Preliminaries}

Exponential dichotomy is at the heart of the fundamental perturbation results for linear systems of Coppel (see [2-3]) and Palmer (see [4-8]), of the spectral theory of Sacker and Sell [9-10], of the geometric theory of Fenichel [11], of perturbation results for invariant manifolds [12], of the fundamental perturbation results for connecting orbits of Beyn and Sandstede (see [13-15]), and it has also proven a formidable ally to justify and gain insight into the behavior of various algorithmic approaches for solving boundary value problems, for approximating invariant surfaces and for computing traveling waves, among other uses (see [16-18]). Using this concept for nonuniform exponential dichotomies case is presented by Barreira, Dragicevic and Valls (see [19-20]).

Theory of dynamic equations on time scales was introduced by Stefan Hilger [21] in order to unify and extend results of differential equations, difference equations, $q$-difference equations, etc. There are many works concerned with dichotomies of dynamic equations on time scales (see [22-24]). In this paper, we want to go further in the exponential dichotomy of dynamic equations. More precisely, we use the method of graph transforms to prove that there exists a unique integral manifold of the exponential dichotomous systems on periodic time scales.

We now introduce some basic concepts of time scales, which can be found in [25-26]. A time scale T is defined as a nonempty closed subset of the real numbers. Define the forward jump operator $\sigma: \mathbb{T} \rightarrow \mathbb{T}$ is defined by $\sigma(t)=\inf \{s \in \mathbb{T}: s>t\}$ and the graininess function $\mu(t)=\sigma(t)-t$ for any $t \in \mathbb{T}$. In the following discussion, the time scale $\mathbb{T}$ is assumed to be unbounded above and below. We have the following several basis definitions (see [25-26]). One has the definition of the exponential function on time scales by

$$
e_{p}(t, s)=\exp \left\{\int_{s}^{t} \xi_{\mu(\tau)}(p(\tau)) \Delta \tau\right\} \quad \text { with } \quad \xi_{h}(z)=\left\{\begin{array}{ll}
z & \text { if } h=0 \\
\log (1+h z) / h & \text { if } h \neq 0
\end{array} .\right.
$$

For any $p \in \mathcal{R}(\mathbb{T}, \mathbb{R})$ and $s, t \in \mathbb{T}$, where log is principal logarithm.

Throughout this paper, we assume that the graininess of the underlying time scale is bounded on $\mathbb{T}^{+}$, i.e., $G=\sup _{t \in \mathbb{T}^{+}} \mu(t)<\infty$. This assumption is equivalent to the fact that there exist positive numbers $m_{1}, m_{2}$ such that for every $t \in \mathbb{T}^{+}$, there exists $c=c(t) \in \mathbb{T}^{+}$satisfying $m_{1} \leqslant c-t<m_{2}$ (also see [27, pp. 319]). We refer [25-26] for more information on analysis on time scales.

We now consider the equation

$$
x^{\Delta}=A(t) x
$$


and the pertubation equations

$$
x^{\Delta}=A(t) x+f(t, x),
$$

where $A \in \mathcal{R}$ and $f(t, x): \mathbb{T} \times \mathbb{R}^{n} \rightarrow \mathbb{R}^{n}$ is $r d$-continuous in the first variable. We also suppose that $f$ satisfies all conditions such that (2) has a unique solution. One can certainly assume that $X(t)$ and $\phi_{t}$ are the families of operators from $\mathbb{R}^{n}$ to $\mathbb{R}^{n}$ such that $X(t) x$ and $\phi_{t}(x)$ are the coresponding solutions of the equations (1) and (2) satisfies $X(0) x=x$ and $\phi_{0}(x)=x$. Throughout this paper, we assume that the graininess of underlying time scale is bounded on $\mathbb{T}$, i.e., $M=\sup \{t: t \in \mathbb{T}\}<\infty$.

Definition 1.1. The equation (1) is said to be bounded growth if there exists constants $N, \omega$ such that

$$
\left\|X(t) X^{-1}(s)\right\| \leqslant N e_{\omega}(t, s) .
$$

For the equations which have bounded growth, we have the following proposition, which is useful later.

Proposition 1.1. Suppose that $f$ is Lipschitz continuous functions in the second argument, i.e. there exists the constants $L$ such that

$$
\|f(t, x)-f(t, y)\| \leqslant L\|x-y\| .
$$

Then

$$
\left\|\left(\phi_{t} \circ \phi_{-s}(x)-X(t) X^{-1}(s) x\right)-\left(\phi_{t} \circ \phi_{-s}(y)-X(t) X^{-1}(s) y\right)\right\| \leqslant N e_{\omega \oplus L N}(t, s)\|x-y\| .
$$

Proof.. First of all, the solution of (2) satisfies the variation of constants formula

$$
\phi_{t} \circ \phi_{-s}(x)=X(t) X^{-1}(s) x+\int_{s}^{t} X(t) X^{-1}(\sigma(\tau)) f\left(\tau, \phi_{\tau} \circ \phi_{-s}(x)\right) d \tau, \quad t \geqslant s .
$$

We have estimate

$$
\begin{aligned}
& \left\|\phi_{t} \circ \phi_{-s}(x)-\phi_{t} \circ \phi_{-s}(y)\right\| \\
\leqslant & \left\|X(t) X^{-1}(s)\right\|\|x-y\|+\int_{s}^{t} \| X(t) X^{-1}(\sigma(\tau))\left(\left\|f\left(\tau, \phi_{\tau} \circ \phi_{-s}(x)\right)-f\left(\tau, \phi_{\tau} \circ \phi_{-s}(x)\right)\right\|\right) d \tau \\
\leqslant & N e_{\omega}(t, s)\|x-y\|+N L \int_{s}^{t} \frac{1}{1+\mu(\tau)} e_{\omega}(t, \tau)\left\|\phi_{\tau} \circ \phi_{-s}(x)-\phi_{\tau} \circ \phi_{-s}(y)\right\| d \tau .
\end{aligned}
$$

This implies

$$
\begin{aligned}
& e_{\ominus \omega}(t, s)\left\|\phi_{t} \circ \phi_{-s}(x)-\phi_{t} \circ \phi_{-s}(y)\right\| \\
& \leqslant N\|x-y\|+N L \int_{s}^{t} e_{\omega}(s, \tau)\left\|\phi_{\tau} \circ \phi_{-s}(x)-\phi_{\tau} \circ \phi_{-s}(y)\right\| d \tau
\end{aligned}
$$

Using the Corollary 6.7 in [25], it reduces that

$$
e_{\ominus \omega}\left\|\phi_{t} \circ \phi_{-s}(x)-\phi_{t} \circ \phi_{-s}(y)\right\| \leqslant N e_{N L}(t, s)\|x-y\| .
$$

The last equation implies the complete proof.

The following concepts almost according to notation in [28] will be needed in our main results.

Definition 1.2. The equation (1) is said to have an exponential dichotomy or to be exponentially dichotomous on $\mathbb{T}$, if there exist a projection matrix $P\left(\right.$ i.e., $\left.P^{2}=P\right)$ on $\mathbb{R}^{n}$ and positive constants $K$ and $\alpha$ such that

$$
\begin{aligned}
& \left\|X(t) P X^{-1}(s)\right\| \leqslant K e_{\ominus \alpha}(t, s), \quad t \geqslant s, \\
& \left\|X(t)(I-P) X^{-1}(s)\right\| \leqslant K e_{\ominus \alpha}(s, t), \quad t \leqslant s .
\end{aligned}
$$


In this paper we only consider the equations (1), which have a projection $P$ satisfies $\left\|X(t) P X^{-1}(t)\right\| \leqslant$ $M, \forall t \in \mathbb{T}$. Let $V_{1}$ and $V_{2}$ be a pair of nonzero subspaces of $\mathbb{R}^{n}$ that are disjoint. We set

$$
\angle\left(V_{1}, V_{2}\right)=\inf \left\{\left\|x_{1}+x_{2}\right\|: x_{i} \in V_{i},\left\|x_{i}\right\|=1, i=1,2\right\}
$$

where the infimum is taken over all pairs of unit vectors belonging to $V_{1}$ and $V_{2}$ respectively. One can easily verify that in this case

$$
\angle\left(V_{1}, V_{2}\right)=2 \sin (\theta / 2),
$$

where $\theta$ is the minimal angle between the subspaces $V_{1}$ and $V_{2}$. In general case we call the quantity $\angle\left(V_{1}, V_{2}\right)$ the angular distance between $V_{1}$ and $V_{2}$.

Definition 1.3. A set $M \subset \mathbb{T} \times \mathbb{R}^{n}$ is said to be an integral manifold of the equation (2) if for every $t \in \mathbb{T}$, we have decomposition $\mathbb{R}^{n}=V_{1}(t) \oplus V_{2}(t)$ such that

$$
\inf _{t \in \mathbb{T}} \angle\left(V_{1}(t), V_{2}(t)\right)>0
$$

and if there exists a family of Lipschitz continuous mapping

$$
g_{t}: V_{1}(t) \rightarrow V_{2}(t), \quad t \in \mathbb{T}
$$

with Lipschitz constants independent of $t$ such that

$$
M=\left\{\left(t, x, g_{t}(x)\right) \in \mathbb{T} \times\left(V_{1}(t) \oplus V_{2}(t)\right): t \in \mathbb{T}, x \in V_{1}(t)\right\}
$$

and

$$
\phi_{t} \circ \phi_{-s}\left(g r\left(g_{s}\right)\right)=g r\left(g_{t}\right), \quad t \geqslant s,
$$

where

$$
\operatorname{gr}\left(g_{t}\right):=\left\{\left(x, g_{t}(x)\right): x \in V_{1}(t)\right\} .
$$

We propose in this paper to show that there exists the unstable integral manifold for the equation (2) with several conditions of the equation (1) and a function $f$. Let $\operatorname{lip}\left(g_{t}\right)$ denote the Lipschitz constant of $g_{t}$. We shall adopt the following metric space

$$
\mathcal{L}(\delta):=\left\{g=\left(g_{t}\right)_{t \in \mathbb{R}} \mid g_{t}: V_{2}(t) \rightarrow V_{1}(t), g_{t}(0)=0, \operatorname{lip}\left(g_{t}\right) \leqslant \delta\right\}
$$

with the metrix

$$
d(g, h):=\sum_{k=1}^{\infty} \frac{1}{2^{k}} \sup _{t \in \mathbb{T},\|x\| \leqslant k}\left\|g_{t}(x)-h_{t}(x)\right\|, \quad g, h \in \mathcal{L}(\delta) .
$$

It is easy to see that $(\mathcal{L}(\delta), d)$ is complete metric space. Under the above notation, we have a following main theorem.

Theorem 1.1. Let (1) has exponential dichotomy on time scale $\mathbb{T}$ which is periodic (see [29]) with the constants $K, \alpha$ and the projection P. Suppose (1) has bounded growth with suitable constants $N, \omega$ (chose later). Then, there exists positive constants $L, \delta$ such that there exists a unique integral manifold $M \subset \mathbb{T} \times \mathbb{R}^{n}$ of equation (2) determined by the family of Lipschitz continuous mappings $\left(g_{t}\right)_{t \in \mathbb{T}} \subset \mathcal{L}(\delta)$ where

$$
g_{t}: V_{2}(t) \rightarrow V_{1}(t)
$$

with $V_{2}(t)=X(t)(I-P) X^{-1}(t) \mathbb{R}^{n}$ and $V_{1}(t)=X(t) P X^{-1}(t) \mathbb{R}^{n}$.

In this paper, we always denote $P(t)=X(t) P X^{-1}(t)$. 


\section{Proof of the Main Theorem}

In this section, we wish to prove the Theorem 1.1. First, we need some following helpful lemmas.

Lemma 2.1. Let $A: \mathbb{R}^{m} \rightarrow \mathbb{R}^{m}$ is an invertible matrix. Assume that $h: \mathbb{R}^{n} \rightarrow \mathbb{R}^{m}$ be a Lipschitz continuous functions with

$$
\operatorname{lip}(h)<\frac{1}{\left\|A^{-1}\right\|}
$$

Then the map $A+h$ is also invertible and that inverse is Lipschitz continuous.

Proof.. See [30-31] for proof.

Lemma 2.2. Under the above notation. For any $h_{0}>0$, if

$$
\delta \leqslant(1+M)\left(K^{-2} e^{\alpha h_{0}}-N e^{(\omega+L N) h_{0}}\right) e^{\alpha h_{0}} \quad \text { and } \quad K^{-2} e^{\alpha h_{0}}>N e^{(\omega+L N) h_{0}}
$$

then for all $g \in \mathcal{L}(\delta)$ and $0 \leqslant t-s \leqslant h_{0}$ we have

$$
(I-P(t)) \phi_{t} \circ \phi_{-s}\left(g_{s}(\cdot)+\cdot\right): V_{2}(s) \rightarrow V_{2}(t)
$$

is a homeomorphism.

Proof.. First, for all $t, s \in \mathbb{T}, t \geqslant s$ we set $h(t, s): V_{2}(s) \rightarrow V_{2}(t)$ is defined

$$
h(t, s) x=(I-P(t)) X(t) X^{-1}(s) g_{s}(x) .
$$

Then, for all $x, y \in V_{2}(s)$, we have the following estimation

$$
\begin{aligned}
\|h(t, s) x-h(t, s) y\| & \leqslant\left\|(I-P(t)) X(t) X^{-1}(s)\left(g_{s}(x)-g_{s}(y)\right)\right\| \\
& \leqslant(1+M)\left\|X(t) X^{-1}(s)\left(g_{s}(x)-g_{s}(y)\right)\right\| \\
& =(1+M)\left\|X(t) P X^{-1}(s)\left(g_{s}(x)-g_{s}(y)\right)\right\| \\
& \leqslant(1+M) K e_{\ominus \alpha}(t, s)\left\|g_{s}(x)-g_{s}(y)\right\| \\
& \leqslant(1+M) K \delta e_{\ominus \alpha}(t, s)\|x-y\|
\end{aligned}
$$

Hence,

$$
\operatorname{lip}(h(t, s)) \leqslant(1+M) K \delta e_{\ominus \alpha}(t, s)
$$

Next, we also have

$$
\begin{aligned}
\left\|\left(\left.X(t) X^{-1}(s)\right|_{\operatorname{KerP}(\mathrm{s})}\right)^{-1}\right\|^{-1} & =\left\|\left.X(s) X^{-1}(t)\right|_{\operatorname{KerP}(\mathrm{t})}\right\|^{-1} \\
& \geqslant K^{-1} e_{\alpha}(t, s) .
\end{aligned}
$$

Finally, let

$$
k(t, s) x=(I-P(t)) \phi_{t} \circ \phi_{-s}(g(x)+x)-(I-P(t)) X(t) X^{-1}(s) x
$$

Then

$$
k(t, s) x=(I-P(t)) \phi_{t} \circ \phi_{-s}(g(x)+x)-(I-P(t)) X(t) X^{-1}(s)\left(g_{s}(x)+x\right)+h(t, s) x .
$$

By Proposition 1.1, one has

$$
\begin{aligned}
\|k(t, s) x-k(t, s) y\| & \leqslant(M+1) \|\left(\phi_{t} \circ \phi_{-s}(g(x)+x)-X(t) X^{-1}(s)\left(g_{s}(x)+x\right)\right) \\
& \left.-\phi_{-s}(g(y)+y)-X(t) X^{-1}(s)\left(g_{s}(y)+y\right)\right)\left\|+(1+M) K \delta e_{\ominus \alpha}(t, s)\right\| x-y \| \\
& \leqslant\left((M+1) N e_{\omega \oplus L N}(t, s)+(1+M) K \delta e_{\ominus \alpha}(t, s)\right)\|x-y\| .
\end{aligned}
$$

Apply the previous lemma again where $h=k(t, s)$ and $A=(I-P(t)) X(t) X^{-1}(s)$ then by hypothesis we obtained the map

$$
(I-P(t)) \phi_{t} \circ \phi_{-s}(g(x)+x): V_{2}(s) \rightarrow V_{2}(t)
$$

is a homeomorphism. 
Lemma 2.3. Under the assumptions and notations above. If $\delta$ satisfies

$$
\delta\|(I-P(t))(x-y)\| \geqslant\|P(t)(x-y)\|
$$

then the mapping $T(t, s)$ with $0 \leqslant t-s \leqslant h_{0}$ given by the formula

$$
\operatorname{gr}\left((T(t, s) g)_{t}\right)=\phi_{t} \circ \phi_{-s}\left(g r\left(g_{s}\right)\right) \in \mathcal{L}\left(\delta^{\prime}\right)
$$

for all $g \in \mathcal{L}(\delta)$ and $t, s \in \mathbb{T}$, is well defined, where

$$
\delta^{\prime}=\frac{\delta K e_{\ominus \alpha}(t, s)+M e_{\omega \oplus L N}(t, s)}{K^{-1} e_{\alpha}(t, s)-(1+M) N e_{\omega \oplus L N}(t, s)} \operatorname{lip}\left((I-P(t)) \phi_{t} \circ \phi_{-s}\left(g_{s}(\cdot)+\cdot\right)\right)
$$

Proof.. We note that

$$
(T(t, s) g)_{t}(x)=P(t) \phi_{t} \circ \phi_{-s}\left(g_{s}(x)+x\right) .
$$

It is necessary and sufficient to show that $(T(t, s) g)_{t}$ are Lipschitz continuous for all $t \in \mathbb{T}$. Indeed, without loss of generality, we assume that $P=\operatorname{diang}(1, \ldots, 1,0, \ldots, 0)$. From the second relation in Definition (1.2) we obtained

$$
\begin{aligned}
\left\|\left.(I-P(t)) X(t) X^{-1}(s)\right|_{\operatorname{Ker} P(s)}\right\| & \geqslant\left\|\left.(I-P(s)) X(s) X^{-1}(t)\right|_{\operatorname{Ker} P(t)}\right\|^{-1} \\
& \geqslant K^{-1} e_{\alpha}(t, s) .
\end{aligned}
$$

Hence, from Lemma 1.1, the last inequality and Definition 2.9 in [32], we have the following estimation

$$
\begin{aligned}
& \left.\|(I-P(t)) \phi_{t} \circ \phi_{-s}(x)-(I-P(t)) \phi_{t} \circ \phi_{-s}(y)\right) \| \\
= & \|\left((I-P(t)) X(t) X^{-1}(s)(I-P(s)) x-(I-P(t)) X(t) X^{-1}(s)(I-P(s)) y\right)+ \\
& (I-P(t))\left(\phi_{t} \circ \phi_{-s}(x)-X(t) X^{-1}(s) x\right)-(I-P(t))\left(\phi_{t} \circ \phi_{-s}(y)-X(t) X^{-1}(s) y\right) \| \\
\geqslant & K^{-1} e_{\alpha}(t, s)\|(I-P(t))(x-y)\|-(1+M) N e_{\omega \oplus L N}(t, s)\|(I-P(t)) x-y\|
\end{aligned}
$$

Because $P(t)$ is projection and (4), with $\delta \leqslant 1 / 2$ we obtain

$$
\|x-y\| \leqslant\|P(t)(x-y)\|+\|(I-P(t))(x-y)\| \leqslant 2 \delta\|(I-P(t))(x-y)\| \leqslant\|(I-P(t))(x-y)\| .
$$

Therefore,

$$
\begin{aligned}
& \left.\|(I-P(t)) \phi_{t} \circ \phi_{-s}(x)-(I-P(t)) \phi_{t} \circ \phi_{-s}(y)\right) \| \\
\geqslant & \left(K^{-1} e_{\alpha}(t, s)-(1+M) N e_{\omega \oplus L N}(t, s)\right)\|(I-P(t))(x-y)\| .
\end{aligned}
$$

On the other hand, by (4) and (6) one has

$$
\begin{aligned}
& \left\|P(t) \phi_{t} \phi_{-s}(x)-P(t) \phi_{t} \phi_{-s}(y)\right\| \\
= & \|\left(P(t) X(t) X^{-1}(s) x-P(t) X(t) X^{-1}(s) y\right)+ \\
& P(t)\left(\phi_{t} \circ \phi_{-s}(x)-X(t) X^{-1}(s) x\right)-P(t)\left(\phi_{t} \circ \phi_{-s}(y)-X(t) X^{-1}(s) y\right) \| \\
\leqslant & K e_{\ominus \alpha}(t, s)\|P(t)(x-y)\|+\|P(t)\| e_{\omega \oplus L N}(t, s)\|x-y\| \\
\leqslant & \delta K e_{\ominus \alpha}(t, s)\|(I-P(t))(x-y)\|+M e_{\omega \oplus L N}(t, s)\|(I-P(t))(x-y)\| \\
\leqslant & \left(\delta K e_{\ominus \alpha}(t, s)+M e_{\omega \oplus L N}(t, s)\right)\|(I-P(t))(x-y)\|
\end{aligned}
$$

Combining (7) and (8), it implies

$$
\begin{aligned}
& \left\|P(t) \phi_{t} \circ \phi_{-s}(x)-P(t) \phi_{t} \circ \phi_{-s}(y)\right\| \\
\leqslant & \frac{\delta K e_{\ominus \alpha}(t, s)+M e_{\omega \oplus L N}(t, s)}{K^{-1} e_{\alpha}(t, s)-(1+M) N e_{\omega \oplus L N}(t, s)}\left\|(I-P(t)) \phi_{t} \circ \phi_{-s}(x)-(I-P(t)) \phi_{t} \circ \phi_{-s}(y)\right\|
\end{aligned}
$$


Hence,

$$
\begin{aligned}
& \left\|P(t) \phi_{t} \circ \phi_{-s}\left(g_{s}(x), x\right)-P(t) \phi_{t} \circ \phi_{-s}\left(g_{s}(y), y\right)\right\| \\
\leqslant & \frac{\delta K e_{\ominus \alpha}(t, s)+M e_{\omega \oplus L N}(t, s)}{K^{-1} e_{\alpha}(t, s)-(1+M) N e_{\omega \oplus L N}(t, s)}\left\|(I-P(t)) \phi_{t} \circ \phi_{-s}\left(g_{s}(x), x\right)-(I-P(t)) \phi_{t} \circ \phi_{-s}\left(g_{s}(y), y\right)\right\| \\
\leqslant & \frac{\delta K e_{\ominus \alpha}(t, s)+M e_{\omega \oplus L N}(t, s)}{K^{-1} e_{\alpha}(t, s)-(1+M) N e_{\omega \oplus L N}(t, s)} \operatorname{lip}\left((I-P(t)) \phi_{t} \circ \phi_{-s}\left(g_{s}(\cdot)+\cdot\right)\right)\|x-y\| .
\end{aligned}
$$

This completes the proof of the lemma.

With the above notation and if the following conditions satisfy

$$
\left\{\begin{array}{l}
\delta^{\prime}=\frac{\delta K e_{\ominus \alpha}(t, s)+M e_{\omega \oplus L N}(t, s)}{K^{-1} e_{\alpha}(t, s)-(1+M) N e_{\omega \oplus L N}(t, s)} \operatorname{lip}\left((I-P(t)) \phi_{t} \circ \phi_{-s}\left(g_{s}(\cdot)+\cdot\right)\right) \leqslant \delta \\
\delta \leqslant(1+M)\left(K^{-2} e^{\alpha h_{0}}-N e^{(\omega+L N) h_{0}}\right) e^{\alpha h_{0}} \quad \text { with } \quad K^{-2} e^{\alpha h_{0}}>N e^{(\omega+L N) h_{0}} \\
M N e_{\omega \oplus L N}(t, s)+K e_{\ominus \alpha}(t, s)+\delta<1 .
\end{array}\right.
$$

Then we have the following proof.

\section{Proof of the main theorem.}

First of all, we prove that $T(t, s)$ is a contraction mapping in $\delta$ when $\delta$ is small enough. Indeed, by Proposition 1.1 and definition of the exponential dichotomy, we get

$$
\begin{aligned}
& \left\|P(t) \phi_{t} \circ \phi_{-s}(x)-P(t) \phi_{t} \circ \phi_{-s}\left(g_{s}((I-P(s)) x)+(I-P(s)) x\right)\right\| \\
\leqslant & \left\|P(t)\left(\phi_{t} \circ \phi_{-s}-X(t) X^{-1}(s)\right)(x)-P(t)\left(\phi_{t} \circ \phi_{-s}-X(t) X^{-1}(s)\right)\left(g_{s}((I-P(s)) x)+(I-P(s)) x\right)\right\| \\
& +\left\|P(t) X(t) X^{-1}(s)(x)-P(t) X(t) X^{-1}(s)\left(g_{s}((I-P(s)) x)+(I-P(s)) x\right)\right\| \\
\leqslant & M N e_{\omega \oplus L N}(t, s)\left\|x-\left(g_{s}((I-P(s)) x)+(I-P(s)) x\right)\right\| \\
& +K e_{\ominus \alpha}(t, s)\left\|x-\left(g_{s}((I-P(s)) x)+(I-P(s)) x\right)\right\| \\
\leqslant & \left(M N e_{\omega \oplus L N}(t, s)+K e_{\ominus \alpha}(t, s)\right)\left\|P(s) x-g_{s}((I-P(s)) x)\right\| .
\end{aligned}
$$

Combining (10), (9) and Lemma 2.3, we get

$$
\begin{aligned}
& \left\|P(t) \phi_{t} \circ \phi_{-s} x-(T(t, s) g)_{t}\left((I-P(t)) \phi_{t} \circ \phi_{-s} x\right)\right\| \\
\leqslant & \left\|P(t) \phi_{t} \circ \phi_{-s} x-P(t) \phi_{t} \circ \phi_{-s}\left(g_{s}((I-P(s)) x)+(I-P(s)) x\right)\right\| \\
& +\| P(t) \phi_{t} \circ \phi_{-s}\left(g_{s}((I-P(s)) x)+(I-P(s)) x\right) \\
& -(T(t, s) g)_{t}\left((I-P(t)) \phi_{t} \circ \phi_{-s} x\right) \| \\
\leqslant & \gamma\left\|P(s) x-g_{s}((I-P(s)) x)\right\|
\end{aligned}
$$

where $\gamma=M N e_{\omega \oplus L N}(t, s)+K e_{\ominus \alpha}(t, s)+\delta<1$ (since $\left.(9)\right)$.

Suppose that $g^{1}, g^{2} \in \mathcal{L}(\delta)$. From the last relation, we obtain

$$
\begin{aligned}
& \left\|\left(T(t, s) g^{1}\right)_{t}(I-P(t)) \phi_{t} \circ \phi_{-s} x-\left(T(t, s) g^{2}\right)_{t}(I-P(t)) \phi_{t} \circ \phi_{-s} x\right\| \\
\leqslant & \gamma\left\|g_{s}^{1}(I-P(s)) x-g_{s}^{2}(I-P(s)) x\right\|
\end{aligned}
$$

for all $x \in \mathbb{R}^{n}$ and $t \in \mathbb{T}$. Thus, for every $n \in \mathbb{N}$ then

$$
\sup _{t \in \mathbb{T},\|x\| \leqslant n}\left\|\left(T(t, s) g^{1}\right)_{t} x-\left(T(t, s) g^{2}\right)_{t} x\right\| \leqslant \gamma \sup _{t \in \mathbb{T},\|x\| \leqslant n}\left\|g_{t}^{1}(x)-g_{t}^{2}(x)\right\| .
$$

Hence $T(t, s)$ is a contraction mapping. 
Supposing that $g$ is the fixed point of $T(t, s)$ (where $\left.0 \leqslant t-s \leqslant h_{0} / 2\right)$ in $\mathcal{L}(\delta)$. Next, we show that $g$ is also the fix point of $T\left(t^{\prime}, s^{\prime}\right)$ for all $t^{\prime}, s^{\prime} \in \mathbb{T}$ and $0 \leqslant t^{\prime}-s^{\prime} \leqslant h_{0} / 2$. Indeed, since $\mathbb{T}$ is periodic, we get $s^{\prime}-t+s, t^{\prime}-t+s, \in \mathbb{T}$ and hence,

$$
T\left(t^{\prime}, s^{\prime}\right) T\left(s^{\prime}, s^{\prime}-t+s\right) g=T\left(t^{\prime}, t^{\prime}-t+s\right) T\left(t^{\prime}-t+s, s^{\prime}-t+s\right) g=T\left(t^{\prime}-t+s, s^{\prime}-t+s\right) g
$$

and

$$
T\left(t^{\prime}, s^{\prime}\right) T\left(s^{\prime}, s^{\prime}-t+s\right) g=T\left(t^{\prime}, s^{\prime}\right) g
$$

From the uniqueness of the fixed point of $T(t, s)$ it implies $T\left(t^{\prime}, s^{\prime}\right) g=g$. This proves theorem.

Acknowledgments. The first author was supported in part by the VNU Project of Vietnam National University No. QG101-15. The authors would also like to extend their appreciations to members of the seminar "Dynamical Systems and Applications" at Hanoi University of Science for helpful suggestions and comments in preparing this paper.

\section{References}

1. N. V. Minh and J. Wu, "Invariant manifolds of partial functional differential equations," J. Differential Equations, vol. 198, no. 2, pp. 381-421, 2004

2. W. A. Coppel, "Stability and Asymptotic Behavior of Differential Equations," Heath Math. Monogr., Heath \& Co., Boston, 1965.

3. W. A. Coppel, "Dichotomies in Stability Theory," Lecture Notes in Math., Springer-Verlag, vol. 629, 1978.

4. K. J. Palmer, "Exponential Dichotomies and Fredholm," Proceedings of the American Mathematical Society, vol. 104, no. 1, pp. 149-156, 1988.

5. K. J. Palmer, "Exponential Dichotomies and Transversal Homoclinic Points," J. Differential Equations, vol. 55, no. 2, pp. 225-256, 1984.

6. K. J. Palmer, "A perturbation theorem for exponential dichotomies," Proc. Roy. Soc. Edinburgh Sect. A, vol. 106, no. 1-2, pp. 25-37, 1987.

7. K. J. Palmer, "Exponential dichotomies for almost periodic equations," Proc. Amer. Math. Soc., vol. 101, no. 2, pp. 293-298, 1987.

8. K. J. Palmer, "Shadowing in Dynamical Systems: Theory and Applications," Kluwer Academic Publishers, Dordrecht, Boston, 2000.

9. R. J. Sacker and G. R. Sell, "A spectral theory for linear differential systems," J. Differential Equations, vol. 27, no. 3, pp. 320-358, 1978.

10. R. J. Sacker and G. R. Sell, "Dichotomies for linear evolutionary equations in Banach spaces," J. Differential Equations, vol. 113, no. 1, pp. 17-67, 1994.

11. N. Fenichel, "Geometric singular perturbation theory for ordinary differential equations," J. Differential Equations, vol. 31, no. 1, pp. 53-98, 1979.

12. N. Fenichel, "Persistence and smoothness of invariant manifolds for flows," Indiana Univ. Math. J., vol. 21, pp. 193-226, 1971.

13. W. Beyn, "On well-posed problems for connecting orbits in dynamical systems," Contemp. Math., vol. 172, pp. 131-168, 1994.

14. W. J. Beyn, "The numerical computation of connecting orbits in dynamical systems," IMA J. Numer. Anal., vol. 10, pp. 379-405, 1990.

15. W. J. Beyn and J. Lorenz, "Stability of traveling waves: Dichotomies and eigenvalue conditions on finite intervals," Numer. Funct. Anal. Optim., vol. 20, pp. 201-244, 1999.

16. U. Ascher, R. M. Mattheij and R. D. Russell, "Solution of Boundary Value Problems for ODEs," Prentice Hall, Englewood Cliffs, NJ, 1988.

17. H. W. Broer, H. M. Osinga and G. Vegter, "Algorithms for computing normally hyperbolic invariant manifolds," Z. Angew. Math. Phys., vol. 48, pp. 480-524, 1997.

18. L. Dieci and J. Lorenz, "Computation of invariant tori by the method of characteristics," SIAM J. Numer. Anal., vol. 32, pp. 1436-1474, 1995.

19. L. Barreira, D. Dragicevic and C. Valls, "Fredholm operators and nonuniform exponential dichotomies," Chaos, Solitons and Fractals, vol. 85, pp. 120-127, 2016.

20. L. Barreira, D. Dragicevic and C. Valls, "Nonuniform exponential dichotomies and Fredholm operators for flows," Aequat. Math., vol. 91, no. 2, pp.301-316, 2017. 
21. S. Hilger, "Analysis on measure chains - a unified approach to continuous and discrete calculus," Results Math., vol. 18, pp. 18-56, 1990.

22. J. Zhang, M. Fan and H. Zhu, "Existence and roughness of exponential dichotomies of linear dynamic equations on time scales," Comp. and Math. with Appl., vol. 59, pp. 2658-2675, 2009.

23. J. Zhang, Y. Song and Z. Zhao, "General exponential dichotomies on time scales and parameter dependence of roughness," Adva. Diff. Equations., 2013.

24. L. Yang, J. Zhang, X. Chang and Z. Liu, "Exponential dichotomy on time scales and admissibility of the pair $\left(C_{r d}^{b}\left(\mathbb{T}^{+}, X\right), L^{p}\left(\mathbb{T}^{+}, X\right)\right)$," Adv. Diff. Equations, 2015.

25. M. Bohner and A. Peterson, "Dynamic equations on time scales: an introduction with applications," Birkhäuser Boston, Inc., 2001.

26. M. Bohner and A. Peterson, "Advances in dynamic equations on time scales," Birkhäuser Boston, Inc., Boston, 2003.

27. C. Pötzsche, "Exponential dichotomies of linear dynamic equations on measure chains under slowly varying coefficients," J. Anal. Math. Appl., vol 289, pp. 317-335, 2004.

28. J. Zhang, M. Fan and H. Zhu, "Necessary and sufficient criteria for the existence of exponential dichotomy on time scales," Comp. and Math. with Appl., vol. 60, pp. 2387-2398, 2010.

29. E. R. Kaufmann and Y. N. Raffoul, "Periodic solutions for a neutral nonlinear dynamical equations on a time scales," J. Math. Anal. Appl., vol. 319, no. 1, pp. 315-325, 2006.

30. R. Martin, "Nonlinear Operators and Differential Equations in Banach Spaces," Wiley Interscience, New York, 1976.

31. Z. Nitecki, "An introduction to the orbit structure of diffeomorphisms," MIT Press, Cambridge, MA, 1971.

32. P. Duarte and S. Klein, "Lyapunov Exponents of Linear Cocycles," Atlantis Press, 2016. 